Journal of Trauma and Acute Care Surgery, Publish Ahead of Print

DOI: $10.1097 /$ TA.0000000000001986

\title{
Increased Trauma Activation Is Not Equally Beneficial For All Elderly \\ Trauma Patients
}

\author{
Bryan W. Carr, MD ${ }^{1}$, Peter M. Hammer, MD ${ }^{1}$, Lava Timsina, PhD, $\mathrm{MPH}^{1}$, \\ Grace Rozycki, MD, MBA ${ }^{1}$, David V. Feliciano, MD $^{2}$, Jamie J. Coleman, MD ${ }^{1}$ \\ ${ }^{1}$ Indiana University School of Medicine \\ ${ }^{2}$ University of Maryland School of Medicine \\ Corresponding author: \\ Jamie J. Coleman, MD \\ jjones79@gmail.com \\ Indiana University School of Medicine \\ 1604 N. Capitol Avenue \\ Office B242 \\ Indianapolis, IN 46202
}

COI statement: No author involved in this study has any conflicts of interest to report

Presented at $31^{\text {ST }}$ Annual Meeting of the Eastern Association for the Surgery of Trauma, January

9-13, 2018 in Orlando, FL

Funding Statement: No funding was received in performance of this project

This is the author's manuscript of the article published in final edited form as:

Carr, B. W., Hammer, P. M., Timsina, L., Rozycki, G., Feliciano, D. V., \& Coleman, J. J. (2018). Increased Trauma Activation Is Not Equally Beneficial For All Elderly Trauma Patients. Journal of Trauma and Acute Care Surgery, Publish Ahead of Print. https://doi.org/10.1097/TA.0000000000001986 


\section{Background}

Physiologic changes in the elderly lead to higher morbidity and mortality after injury. Increasing level of trauma activation has been proposed to improve geriatric outcomes; but, the increased cost to the patient and stress to the hospital system are significant downsides. The purpose of this study was to identify the age at which an increase in activation status is beneficial.

\section{Methods}

A retrospective review of trauma patients $\geq 70$ years old from October 1,2011 , to October 1 , 2016 was performed. On October 1, 2013, a policy change increased the activation criteria to the highest level for patients $\geq 70$ years of age with a significant mechanism of injury. Patients who presented prior to (PRE) were compared to those after the change (POST). Data collected included age, injury severity score (ISS), length of stay (LOS), complications and mortality. Primary outcome was mortality and secondary outcome was LOS. Multivariable regressions controlled for age, ISS, injury mechanism, and number of complications.

Results

4341 patients met inclusion criteria, 1919 in PRE and 2422 in POST. Mean age was 80.4 and 81 years in PRE and POST groups respectively $(\mathrm{p}=0.0155)$. Mean ISS values were 11.6 and 12.4 ( $p<0.0001)$ for the PRE and POST groups. POST had more level 1 activations (696 vs. 220, $\mathrm{p}<0.0001)$. After controlling for age, ISS, mechanism of injury, and number of complications, mortality was significantly reduced in the POST group $\geq$ age 77 years (OR $0.53,95 \%$ CI: 0.3 0.87), (Figure 1). Hospital LOS was significantly reduced in the POST group $\geq$ age 78 (regression coefficient -0.55, 95\% CI: -1.09 , -0.01) (Figure 2). 


\section{Conclusions}

This study suggests geriatric trauma patients $\geq 77$ years benefit from the highest level of trauma activation with shorter LOS and lower mortality. A focused approach to increasing activation level for elderly patients may decrease patient cost.

Level of Evidence: Level III

Type of Study: Economic/Decision

Keywords: geriatric trauma, trauma activation criteria, triage 


\section{Background}

The elderly are a steadily expanding population of patients. Today's population involves a larger proportion of elderly people who are projected to live longer and are involved in more active lifestyles. The life expectancy of a 65 year old man or woman is approximately 18 and 20 years respectively compared to approximately 14 and 18 years in 1980 (1). It is currently estimated that by 2030 , approximately $21 \%$ of the population in the United States will be over the age of 65(2). In correlation with this aging of the United States population is an increase in the overall number and proportion of injured elderly patients presenting to trauma centers. This is reflected in data from the National Trauma Data Bank, which revealed an increase from 23\% of patients aged 65 and older presenting to Level I and Level II trauma centers in 2003 to $30 \%$ in 2009(3). The increasing age of trauma patients has forced providers to focus on the physiologic changes, rehabilitation requirements, and social needs of this patient population.

Elderly trauma patients not only present a challenge due to their increasing numbers. Mortality in patients over the age of 65 has been shown to be at least twice that of younger patients with equivalent injury severity(4). Many factors have been associated with this increased mortality and include: polypharmacy, more comorbid conditions, higher risk for malnutrition, and less physiologic reserve compared to younger patients (5-13).

These physiologic differences are often not accounted for in standard triage criteria and have consistently led to the under triage of trauma patients over the age of $60(7,14-17)$. An increase in trauma activation for elderly patients has been proposed by several authors, however, the age at which a trauma patient becomes "elderly" remains uncertain, with ages suggested ranging from 55 to $80(7,13,17,18)$. Depending upon the age criterion used, this could result in a large increase in trauma team activations, place an undue burden upon trauma systems, and 
dramatically increase the cost of care for elderly patients. The aim of this study is to identify an age cutoff that conveys a mortality benefit in efforts to better optimize the resource and cost utilization of trauma systems.

\section{Methods}

After approval was obtained by the Institutional Review Board of Indiana University School of Medicine, the records of trauma patients over the age of 70 presenting to the emergency department of Indiana University Health (IUH) Methodist Hospital from October 1, 2011, to October 1, 2016, were retrospectively reviewed. IUH Methodist Hospital, an American College of Surgeons Level I Trauma Center, is the state's largest hospital and busiest trauma center, with approximately 3,600 trauma admissions per year.

There are four levels of trauma team activation at IUH Methodist Hospital. Patients are triaged based on prehospital reports and according to published criteria (Table 1). The highest level, Level I activation, requires immediate evaluation from the entire trauma team which includes: attending trauma surgeon, general surgery residents, the emergency department (ED) attending physician and resident, 3 ED nurses, a clinical specialty pharmacist, and representatives from chaplaincy and social services. Level II activation requires prompt evaluation by an attending trauma surgeon, general surgery resident, ED resident, and ED attending physician. Level III activations are evaluated by the ED physicians alone; the trauma team is consulted on an as needed basis. The Level IV designation includes patients that have single organ system injuries for which the inpatient trauma service was never consulted.

Criteria for Level I activation was expanded on October 1, 2013 to include all patients aged greater than 70 years with Level II activation criteria. A retrospective review of all trauma 
patients aged 70 years or older admitted from October 1, 2011 through October 1, 2016 was performed. Patients admitted to the trauma service before October 1, 2013 were designated the PRE group, and those admitted after were designated the POST group. Exclusion criteria were pre-hospital cardiac arrest, death in the ED, and burn injury. Data were collected from the IUH Methodist Hospital trauma registry and included patient age, mechanism of injury, injury severity score (ISS), ED length of stay (LOS), hospital LOS, total number of in hospital complications, mortality, and discharge destination.

The primary outcome was mortality, and secondary outcome was hospital LOS. Statistical analysis was performed using Stata SE 14.2. Multivariable regression analysis was performed to control for age, mechanism of injury, ISS, and number of complications. Logistic regression was used to estimate the adjusted odds ratio (95\% confidence interval (CI)) to examine the association of age with mortality. Linear regressions parameter $(95 \% \mathrm{CI})$ was used to examine the association of age with hospital LOS. For all significant tests, $<0.05$ was used to determine significance. Over and under-triage rates were calculated using the Cribari Method, and the difference in these rates were compared with a Mann-Whitney U Test.

\section{Results}

A total of 4,341 patients met criteria for inclusion, with 1,919 patients in the PRE group and 2,422 in the POST group (Table 2). The mean age was 80.4 and 81 years in the PRE and POST groups respectively ( $\mathrm{p}=0.0155)$. Mean ISS values were 11.6 and $12.4(\mathrm{p}<0.0001)$ for the PRE and POST groups. Mechanism of injury was $67.3 \%$ blunt, $1.26 \%$ penetrating, and $31.4 \%$ other mechanism for the PRE group and $98.9 \%$ blunt, $0.78 \%$ penetrating, and $0.29 \%$ other mechanism for the POST group $(\mathrm{p}<0.0001)$. Length of stay was similar between groups: 5.9 
days for PRE and 5.8 days for POST ( $\mathrm{p}=0.45$ ). For the PRE group, $82.9 \%$ had no complications, $14.6 \%$ had 1 to 2 complications, and $2.5 \%$ had 3 or more; in the POST group, $73.2 \%$ had no complications, $23.5 \%$ had $1-2$ complications, and $3.26 \%$ had 3 or more $(\mathrm{p}<0.001)$. Mortality was similar between groups: $8 \%$ in PRE and $7.1 \%$ in POST $(\mathrm{p}=0.52)$. After adjusting for injury mechanism, LOS and number of complications there were no statistically significant differences in age $(\mathrm{p}=0.053)$ or ISS $(\mathrm{p}=0.820)$. Levels of trauma activation between groups were significantly different: level one activations increased in the POST group (220 PRE vs. 696 POST), level 2 activations decreased (768 PRE vs. 495 POST), level 3 activations stayed similar (165 PRE vs. 150 POST), and level 4 activations were higher in the POST group (766 PRE vs. 1081 POST) $(\mathrm{p}<0.001)$. If level 4 patients were disregarded, the difference in level of activation remained significant $(\mathrm{p}<0.001)$.

After controlling for age, ISS, mechanism of injury, and number of complications, in hospital mortality was significantly reduced in the POST group at age 77 years (OR 0.53, 95\% CI: $0.3-0.87, \mathrm{p}=0.011$ ), (Figure 1). This mortality reduction for level one trauma activation held for all subsequent ages after 77 . Prior to age 77 , there was not a statistically significant mortality benefit associated with higher level of trauma activation. Hospital LOS was significantly reduced in the POST group at age 78 and older (regression coefficient $-0.55,95 \%$ CI: -1.09 , 0.01, $\mathrm{p}=0.048$ ) (Figure 2). Additionally, the LOS benefit remained significant for all subsequent ages after age 78 . Prior to age 78 there was not a statistically significant reduction in hospital LOS between the PRE and POST groups. The over-triage rate in the PRE group was $47.03 \%$ compared to $51.96 \%$ in the POST group; this difference did not reach statistical significance $(\mathrm{p}=0.629)$. The undertriage rates between groups were not significantly different: $28.51 \%$ in the PRE group vs. $26.26 \%$ in the POST group ( $\mathrm{p}=0.4)$. 


\section{Discussion}

It is well-recognized that care of elderly patients who have been traumatically injured is challenging, complex, and increasingly significant to trauma centers secondary to the increase of this population. With the geriatric population steadily growing, traumatic injuries in this population will continue to be an expanding issue faced by every trauma surgeon. These patients have repeatedly demonstrated that advanced age, increased comorbidities, reduced physiologic reserve, and presence of frailty lead to poor outcomes after injury (10). Attempting to establish effective interventions for this population constantly challenges trauma systems.

Many studies have sought to improve outcomes for this patient population and several have focused on pre-hospital triage criteria and post-admission management strategies $(6,7,11$, 19-25). Current literature has examined the development of prognostic tools, early nutritional assessment, early involvement of specialty services such as palliative medicine or geriatricians, and geriatric specific trauma protocols and all have been shown to reduce adverse outcomes to some degree $(5,9,26,27)$. Our institution recently published data on age as a criterion for increasing the level of trauma activation (28). Higher level of activation correlated with shorter emergency department (ED) length of stay (LOS) and mortality for patients over the age of 70 (28). Although it is used as one of the most important prognosticators, age is unable to fully account for the mortality risk within this population. Campbell-Furtick et. al. recently associated, via multi-variate logistic regression in a stepwise fashion, only a $10 \%$ contribution of age to overall post-trauma mortality. Within this cohort of 872,861 patients, the straight line trends in the data showed increases in mortality at ages 37, 60, and 78 (12). However, the age at which a trauma patient becomes "elderly" remains uncertain, and no consensus has yet been established. 
The age of 70 has been shown to have an association with substantial mortality despite minor injuries (7). Additionally, ISS, shock index, and admission systolic blood pressure have been linked to predicting mortality in this population $(8,23)$. A recent study demonstrated that age may only contribute in a minor fashion when looking at cause of mortality (12) but, alternatively, it remains the fastest way to stratify and identify the "geriatric" trauma patient. Due to these advantages of using age as a means of improving outcomes, expanding trauma activation criteria to include age has been studied $(7,28,29)$. The goals of this strategy have been to provide timely evaluation, diagnosis, and resource allocation by expanding the trauma activation criteria for the highest level to include age. However, this change can result in a significant increase in level 1 activations compared to traditional methods potentially placing an undue financial burden on patients and negatively impact the utilization of resources within the trauma system.

Our study sought to identify at what age this intervention was most beneficial to the patient and reduce mortality. We identified no difference in mortality or hospital LOS when Level I trauma activation was initiated prior to age 77 and 78 respectively. Early evaluation, diagnostic testing, disposition planning, and hospital admission that comes in conjunction with Level I activation does not benefit all patients equally. There is a mortality benefit to Level I activation after age 70 (28), but, within that decade this is seen only after the age of 77 . In this study, 1,427 patients (32.9\%) of patients were under the age of 77 .

More level I activations subsequently places a significant burden on the trauma service, system, and resources available. For patient charges in our system, a Level I trauma activation carries a total charge of $\$ 21,326$ as compared to $\$ 15,354$ for a Level II activation. There were 1,645 (37.9\%) patients who were aged 70 to 77 years old. These patients otherwise met Level II 
criteria, and allowing them to be evaluated as such could alleviate this increased system burden without a concomitant increase in mortality or LOS. If this group is evaluated as Level II trauma, the savings to the patients would have been $\$ 9,823,940$.

The Eastern Association for the Surgery of Trauma (EAST) Guidelines on geriatric trauma state clearly that patients aged 65 or older with pre-existing comorbid conditions or severe anatomic injuries should be treated in a trauma center (20). Not all trauma centers may be equal in caring for this population. Overall volume of geriatric trauma patients cared for is another important aspect to improving outcomes for this cohort; centers whose patients are proportionally older have been shown to reduce overall complications from $22 \%$ to $14 \%$, reduce failure to rescue from $4 \%$ down to $2 \%$, and overall mortality (30). Tracking age alone estimated a $16 \%$ mortality for patients aged 70 years and above with minor injuries, and up to $50 \%$ for major injuries (7). These data have led trauma centers to alter both their triage criteria as well as post-injury quality improvement efforts to best optimize the patients' care. Without the ability to change our patient demographics, our center changed the Level I activation criteria to include age 70 or greater in efforts to improve care delivery. Our results suggest that this age cutoff may not offer the mortality benefit equally, and a more focused approach to increasing level of activation can lead to similar mortality benefit without increasing LOS or overall complication rates.

Broadening the criteria for trauma activation does raise concerns for over-triage. Our previous study evaluated this point, and only $2 \%$ of the over-triaged patients within this time frame were older than 70 years old (28). Furthermore, our data did not show an increase in overtriage, nor a decrease in under-triage, between groups with this policy change. Under-triage of the geriatric trauma patient is well established, and has been reported to be as high as $61 \%$ 
nationally. Risk factors for under-triage in this study included age, female gender, and fall related injuries (15). This holds true for both EMS personnel as well as non-trauma centers who do not initiate inter-facility transfers to tertiary trauma centers for more severely injured older adults (14). We did not see any significant increase in over-triage based on our criteria modification, and it may have allowed a more timely evaluation by the trauma service in a previously under-triaged patient despite no significant decrease in undertriage.

This study is not without limitations. These patients were evaluated retrospectively, and causality is unable to be established. Age alone is not going to be the specific cause of increased mortality in this patient population. Other variables could be contributing to the differences that we have observed. Comorbidities and frailty were not directly measured despite frailty seemingly superior to age when discussing outcomes for this cohort (10). However, this information is not readily available to prehospital providers or trauma surgeons in the emergency department. Any evaluation of frailty would take more time to assess compared to age alone, therefore initial interventions are directed at rapidly assessed patient information. Some patients were excluded due to missing data for the collected variables, but the number was low and evenly distributed across the PRE and POST groups. The under and over-triage rates may not be clinically accurate. The Cribari method was used to identify both under and over-triaged patients and does not take into account any of the activation criteria, but rather uses ISS alone and level of activation. Lastly, these data were collected at the index hospital admission alone, and no follow up data was recorded, so long term outcomes cannot be commented on for this study.

Using age alone to enact a simple intervention of higher level of trauma activation reduces mortality in injured, geriatric trauma patients. This also applies a significant pressure on the trauma system for resource utilization and increases the costs to the patient. Using a more 
focused approach, this mortality benefit was not significant until age 77, and the reduced length of stay benefit was significant at age 78 without any changes in complication rate. These data suggest that tightening the activation criteria to a later age could offload a portion of the burden to the system. 


\section{Individual Author Contributions}

Carr: literature search, study design, data collection, data analysis, data interpretation, writing

Hammer: literature search, study design, data collection

Timsina: data analysis, data interpretation

Rozycki: study design, critical revision

Feliciano: study design, critical revision

Coleman: literature search, study design, data interpretation, writing, critical revision 


\section{References:}

1. Bell FC, Miller ML. Life Tables for the United States Social Security Area 1900-21002005 8/17/2017 [cited 2017. Available from: https://www.ssa.gov/oact/NOTES/as120/LifeTables _Body.html.

2. Statistics FIFoA-R. Older Americans 2012: Key Indicators of Well-Being. Washington, D.C.; 2012.

3. Kozar RA, Arbabi S, Stein DM, Shackford SR, Barraco RD, Biffl WL, Brasel KJ, Cooper Z, Fakhry SM, Livingston D, et al. Injury in the aged: Geriatric trauma care at the crossroads. $J$ Trauma Acute Care Surg. 2015;78(6):1197-209.

4. Perdue PW, Watts DD, Kaufmann CR, Trask AL. Differences in mortality between elderly and younger adult trauma patients: geriatric status increases risk of delayed death. J Trauma. 1998;45(4):805-10.

5. Cook AC, Joseph B, Inaba K, Nakonezny PA, Bruns BR, Kerby JD, Brasel KJ, Wolf SE, Cuschieri J, Paulk ME, et al. Multicenter external validation of the Geriatric Trauma Outcome Score: A study by the Prognostic Assessment of Life and Limitations After Trauma in the Elderly (PALLIATE) consortium. J Trauma Acute Care Surg. 2016;80(2):204-9.

6. Demetriades D, Karaiskakis M, Velmahos G, Alo K, Newton E, Murray J, Asensio J, Belzberg H, Berne T, Shoemaker W. Effect on outcome of early intensive management of geriatric trauma patients. Br J Surg. 2002;89(10):1319-22.

7. Demetriades D, Sava J, Alo K, Newton E, Velmahos GC, Murray JA, Belzberg H, Asensio JA, Berne TV. Old age as a criterion for trauma team activation. J Trauma. 2001;51(4):7546; discussion 6-7. 
8. Hashmi A, Ibrahim-Zada I, Rhee P, Aziz H, Fain MJ, Friese RS, Joseph B. Predictors of mortality in geriatric trauma patients: a systematic review and meta-analysis. $J$ Trauma Acute Care Surg. 2014;76(3):894-901.

9. Muller FS, Meyer OW, Chocano-Bedoya P, Schietzel S, Gagesch M, Freystaetter G, Neuhaus V, Simmen HP, Langhans W, Bischoff-Ferrari HA. Impaired nutritional status in geriatric trauma patients. Eur J Clin Nutr. 2017;71(5):602-6.

10. Joseph B, Pandit V, Zangbar B, Kulvatunyou N, Hashmi A, Green DJ, O'Keeffe T, Tang A, Vercruysse G, Fain MJ, et al. Superiority of frailty over age in predicting outcomes among geriatric trauma patients: a prospective analysis. JAMA surgery. 2014;149(8):766-72.

11. Bradburn E, Rogers FB, Krasne M, Rogers A, Horst MA, Beelen MJ, Miller JA. High-risk geriatric protocol: improving mortality in the elderly. J Trauma Acute Care Surg. 2012;73(2):435-40.

12. Campbell-Furtick M, Moore BJ, Overton TL, Laureano Phillips J, Simon KJ, Gandhi RR, Duane TM, Shafi S. Post-trauma mortality increase at age 60: a cutoff for defining elderly? Am J Surg. 2016;212(4):781-5.

13. Goodmanson NW, Rosengart MR, Barnato AE, Sperry JL, Peitzman AB, Marshall GT. Defining geriatric trauma: when does age make a difference? Surgery. 2012;152(4):668-74; discussion 74-5.

14. Garwe T, Stewart K, Stoner J, Newgard CD, Scott M, Zhang Y, Cathey T, Sacra J, Albrecht RM. Out-of-hospital and Inter-hospital Under-triage to Designated Tertiary Trauma Centers among Injured Older Adults: A 10-year Statewide Geospatial-Adjusted Analysis. Prehosp Emerg Care. 2017:1-10. 
15. Kodadek LM, Selvarajah S, Velopulos CG, Haut ER, Haider AH. Undertriage of older trauma patients: is this a national phenomenon? J Surg Res. 2015;199(1):220-9.

16. Newgard CD, Richardson D, Holmes JF, Rea TD, Hsia RY, Mann NC, Staudenmayer K, Barton ED, Bulger EM, Haukoos JS, et al. Physiologic field triage criteria for identifying seriously injured older adults. Prehosp Emerg Care. 2014;18(4):461-70.

17. Nakamura Y, Daya M, Bulger EM, Schreiber M, Mackersie R, Hsia RY, Mann NC, Holmes JF, Staudenmayer K, Sturges Z, et al. Evaluating age in the field triage of injured persons. Ann Emerg Med. 2012;60(3):335-45.

18. Caterino JM, Valasek T, Werman HA. Identification of an age cutoff for increased mortality in patients with elderly trauma. Am J Emerg Med. 2010;28(2):151-8.

19. Bar-Or D, Salottolo KM, Orlando A, Mains CW, Bourg P, Offner PJ. Association between a geriatric trauma resuscitation protocol using venous lactate measurements and early trauma surgeon involvement and mortality risk. J Am Geriatr Soc. 2013;61(8):1358-64.

20. Calland JF, Ingraham AM, Martin N, Marshall GT, Schulman CI, Stapleton T, Barraco RD, Eastern Association for the Surgery of T. Evaluation and management of geriatric trauma: an Eastern Association for the Surgery of Trauma practice management guideline. J Trauma Acute Care Surg. 2012;73(5 Suppl 4):S345-50.

21. Callaway DW, Shapiro NI, Donnino MW, Baker C, Rosen CL. Serum lactate and base deficit as predictors of mortality in normotensive elderly blunt trauma patients. $J$ Trauma. 2009;66(4):1040-4.

22. Davis JW, Kaups KL. Base deficit in the elderly: a marker of severe injury and death. $J$ Trauma. 1998;45(5):873-7. 
23. Pandit V, Rhee P, Hashmi A, Kulvatunyou N, Tang A, Khalil M, O'Keeffe T, Green D, Friese RS, Joseph B. Shock index predicts mortality in geriatric trauma patients: an analysis of the National Trauma Data Bank. J Trauma Acute Care Surg. 2014;76(4):1111-5.

24. Scalea TM, Simon HM, Duncan AO, Atweh NA, Sclafani SJ, Phillips TF, Shaftan GW. Geriatric blunt multiple trauma: improved survival with early invasive monitoring. J Trauma. 1990;30(2):129-34; discussion 34-6.

25. Brown JB, Gestring ML, Forsythe RM, Stassen NA, Billiar TR, Peitzman AB, Sperry JL. Systolic blood pressure criteria in the National Trauma Triage Protocol for geriatric trauma: 110 is the new 90. J Trauma Acute Care Surg. 2015;78(2):352-9.

26. Olufajo OA, Tulebaev S, Javedan H, Gates J, Wang J, Duarte M, Kelly E, Lilley E, Salim A, Cooper Z. Integrating Geriatric Consults into Routine Care of Older Trauma Patients: OneYear Experience of a Level I Trauma Center. J Am Coll Surg. 2016;222(6):1029-35.

27. Kupensky D, Hileman BM, Emerick ES, Chance EA. Palliative Medicine Consultation Reduces Length of Stay, Improves Symptom Management, and Clarifies Advance Directives in the Geriatric Trauma Population. J Trauma Nurs. 2015;22(5):261-5.

28. Hammer PM, Storey AC, Bell T, Bayt D, Hockaday MS, Zarzaur BL, Jr., Feliciano DV, Rozycki GS. Improving geriatric trauma outcomes: A small step toward a big problem. $J$ Trauma Acute Care Surg. 2016;81(1):162-7.

29. Kalina M. Implementation of a Trauma Service Activation and Admission Policy for Very Elderly Trauma Patients: Impact on Hospital Efficiency and Patient Outcomes. Am Surg. 2016;82(6):493-6.

30. Bukur M, Simon J, Catino J, Crawford M, Puente I, Habib F. The G60 Trauma Center: A Future Consideration? Am Surg. 2017;83(6):547-53. 
Figure Legends:

Figure 1: OR < 1 indicates patients treated in the POST October 1, 2013 group were significantly less likely to die compared to PRE period. 95\% CI depicted surrounding each OR.

Figure 2: Coeff $<0$ indicates patients treated in the POST October 1, 2013 group had significantly shorter hospital LOS compared to the PRE group. 95\% CI depicted surrounding each Coeff. 
Figure 1

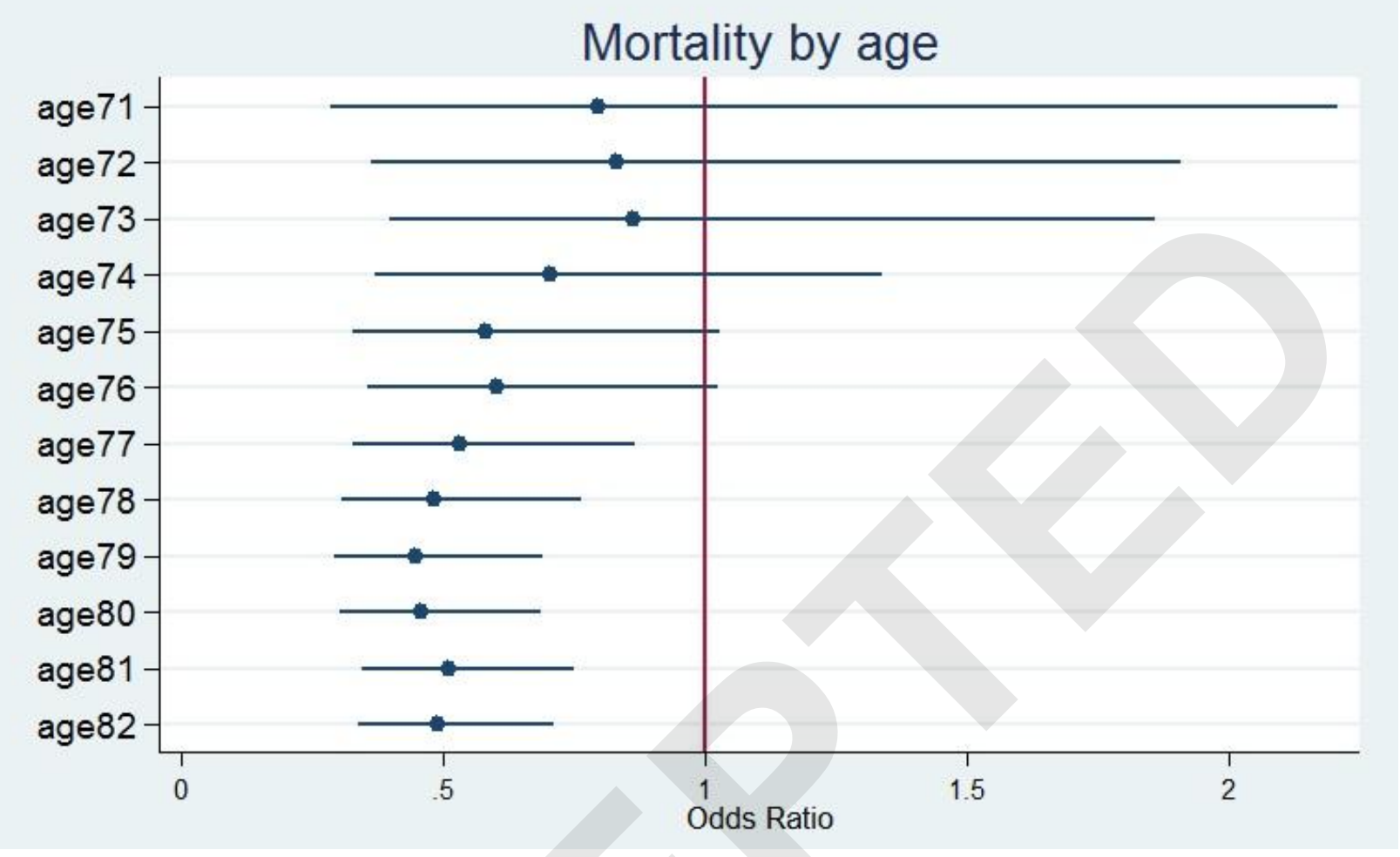


Figure 2

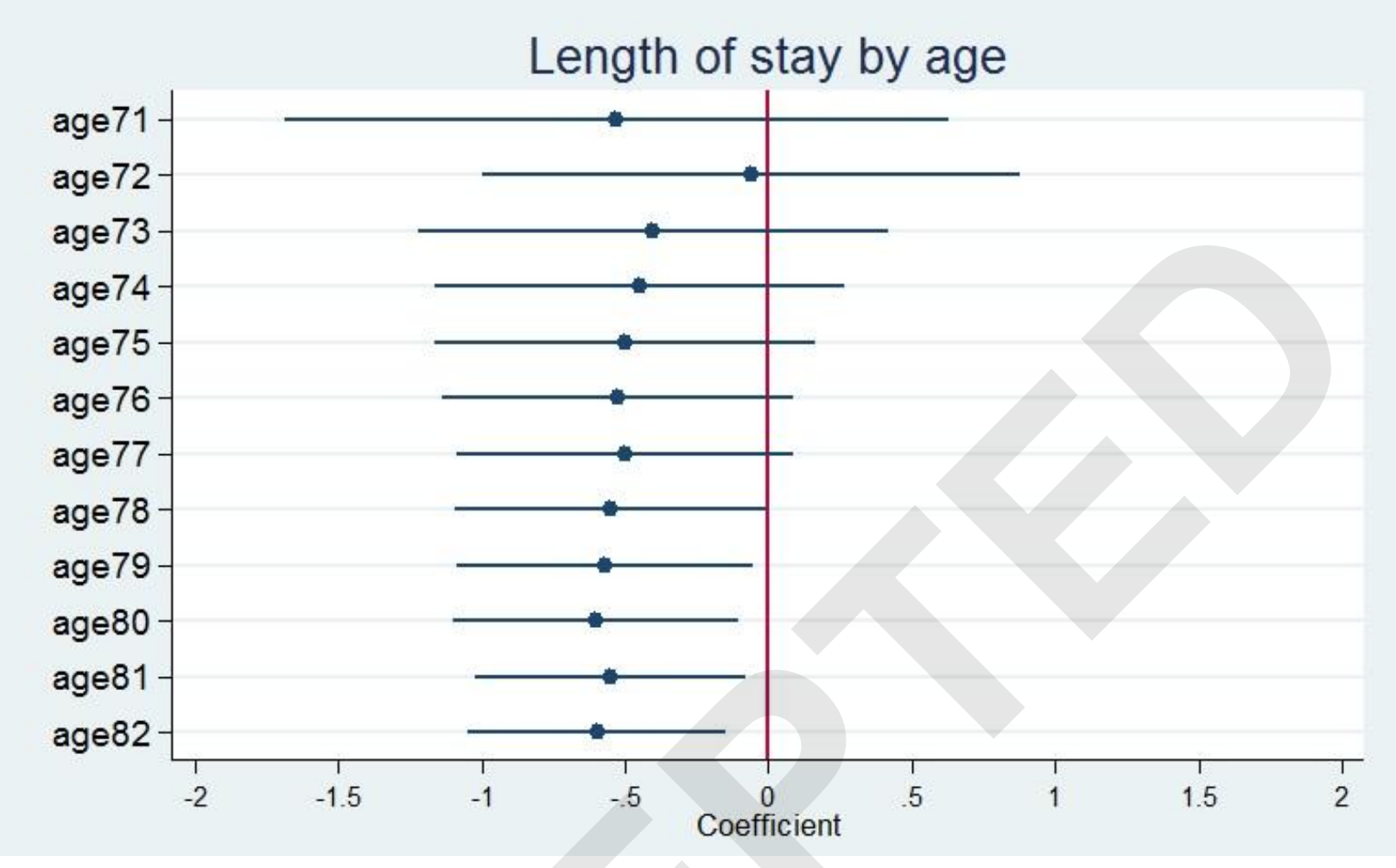


Table 1:

Trauma Activation Criteria

Level 1

Activated if any 1 of these criteria are met:

Arrives with tracheal Intubation

$\mathrm{RR}<10$ or $>29$

Infant $<1$ yr with $R R<20$

SBP $<90$

Receiving blood transfusion during transfer

GCS $<14$

Open or depressed skull fracture

Paralysis

Penetrating Injury to head, neck, or torso

Penetrating extremity injury proximal to elbow

Penetrating extremity injury proximal to knee

Flail chest

$\geq 2$ long bong fractures

Crushed, degloved, or mangled extremity

Amputation proximal to wrist or ankle

Pelvic fracture

Pregnancy $>20$ weeks with c/o vaginal bleeding or abdominal/pelvic pain

*Age $\geq 70$ with Trauma 2 Activation Criteria*

Physician discretion

\section{Level 2}

Activated if any 1 of these criteria are met:

Fall $>20^{\prime}$

MVC with $>12$ " intrusion on occupant's side

MVC with prolonged extrication

MVC with $>18$ " intrusion on any side

Ejection from vehicle

Rollover MVC

Death of a passenger in same vehicle

Vehicle telemetry data consistent with high risk of injury

Pedestrian vs. vehicle who was thrown, run over, or significant impact (>20 mph)

Bicyclist vs. vehicle who was thrown, run over, or significant impact ( $>20 \mathrm{mph}$ )

Motorcycle crash at $>20 \mathrm{mph}$

Child with fall $>10$ feet or 2-3 times the child's height

EMT or paramedic judgement 


\begin{tabular}{|c|c|c|c|c|}
\hline & & PRE & POST & $\mathrm{p}$ \\
\hline \multicolumn{2}{|l|}{$n$} & 1919 & 2422 & \\
\hline \multicolumn{2}{|l|}{ Age } & 80.4 & 81 & 0.0155 \\
\hline \multicolumn{2}{|l|}{ ISS } & 11.58 & 12.45 & 0.001 \\
\hline \multicolumn{2}{|l|}{ LOS (days) } & 5.9 & 5.8 & 0.45 \\
\hline \multirow{3}{*}{$\mathrm{MOI}$} & Blunt (\%) & 67.3 & 98.9 & \multirow{3}{*}{$<0.001$} \\
\hline & Penetrating(\%) & 1.26 & 0.78 & \\
\hline & Other (\%) & 31.4 & 0.29 & \\
\hline \multirow{3}{*}{ Number of Complications } & None (\%) & 82.9 & 73.2 & \multirow{3}{*}{$<0.001$} \\
\hline & $1-2(\%)$ & 14.6 & 23.5 & \\
\hline & $3+(\%)$ & 2.5 & 3.26 & \\
\hline \multicolumn{2}{|l|}{ Mortality (\%) } & 8 & 7.1 & 0.252 \\
\hline \multirow[t]{4}{*}{ Level of Activation } & Level 1 & 220 & 696 & \multirow{4}{*}{$<0.001$} \\
\hline & Level 2 & 768 & 495 & \\
\hline & Level 3 & 165 & 150 & \\
\hline & Level 4 & 766 & 1081 & \\
\hline
\end{tabular}

